\begin{tabular}{l|l|l|l|l} 
Revue suisse Zool. & Tome 81 & Fasc. 2 & p. 387-395 & Genève, juin 1974
\end{tabular}

\title{
Ragni d'Italia XXI. \\ Settimo contributo alla conoscenza dei ragni cavernicoli di Sardegna e descrizione di una nuova specie di Corsica (Araneae)
}

di

\section{Paolo Marcello BRIGNOLI}

Con 9 figure nel testo

Durante un loro breve soggiorno in Sardegna i ben noti biospeleologi ginevrini Prof. Dr. V. Aellen e Dr. P. Strinati visitarono tre grotte raccogliendo un certo numero di ragni tra cui anche un maschio di Tegenaria henroti Dresco, 1956. L'esame di questo individuo mi ha permesso di appurare che il materiale della grotta di S. Giovanni di Domusnovas da me a suo tempo (1971) attribuito a $T$. henroti non va ascritto a questa specie, ma bensì ad una nuova che qui descrivo.

All'elenco di questo materiale sardo ho ritenuto opportuno aggiungere la descrizione di un'altra nuova Tegenaria della Corsica di cui alcuni individui erano conservati nel fondo generale del Museo di Ginevra.

Ringrazio vivamente, oltre ai signori Aellen e Strinati, anche il Dr. B. Hauser che mi ha affidato in istudio il materiale che ha dato spunto a questo studio e che è conservato nelle collezioni del Muséum d'histoire naturelle de Genève.

\section{MATERIALE DI SARDEGNA}

\section{Fam. Leptonetidae}

\section{Leptoneta patrizii Roewer 1953}

Grotta di S. Giovanni, 81 Sa/CA, Domusnovas, 22..3.71, V. Aellen \& P. Strinati leg., 1 ㅇ, 1 ○. 
Specie nota finora solo di questa grotta ove, a quanto pare, è piuttosto comune. Qualche anno fa l'ho ridescritta sui tipi (BRIGNOLI, 1969).

\section{Fam. Pholcidae}

Pholcus phalangioides (Fuesslin) 1775

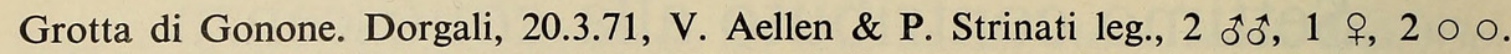
Grotta Verde, $3 \mathrm{Sa} / \mathrm{SS}$, Alghero, 18.3.71, V. Aellen \& P. Strinati leg., 3 웅.

Già nota di parecchie grotte di Sardegna.

\section{Fam. Theridiidae}

Pholcomma gibbum (Westring) 1851

Grotta di S. Giovanni, 81 Sa/CA, Domusnovas, 22.3.71, V. Aellen \& P. Strinati leg., 1 ‥

Già nota epigea di Sardegna (GARNERI, 1902); non era stata ancora trovata in grotte dell'isola; è però citata di cavità del Trentino e della Sicilia (cfr. BRIGNOLI, 1972).

Steatoda grossa (C. L. Koch) 1838

Grotta Verde, 3 Sa/SS, Alghero, 18.3.71, V. Aellen \& P. Strinati leg., 1 ㅇ, $2 \circ \circ$.

Comune in tutta l'Italia; già nota di grotte di Puglie, Calabria, Sicilia e Sardegna (103 Sa/NU; cfr. BRIGNOLI, 1972).

\section{Fam. Agelenidae}

Tegenaria henroti Dresco 1956

Grotta di Gonone, Dorgali, 20.3.71, V. Aellen \& P. Strinati leg., 1 ô.

Ridescrizione - $\hat{\sigma}$ ( $(+$ tuttora ignota): prosoma bruniccio senza peculiarità ; occhi anteriori in una fila a leggera concavità posteriore, occhi posteriori in una fila diritta; LA : LP/MP : MA $=10: 8: 6$; intervalli tra gli anteriori pari alla metà del diametro dei $\mathrm{MA}$, intervalli tra i posteriori pari al diametro dei MA. Labium quadrangolare, più lungo che largo; sterno unicolore, bruniccio, cordiforme con piccola punta separante le IV coxe meno del loro diametro. Cheliceri robusti, non genicolati, con 5/6-4 denti (5/6 al margine inferiore, 6 a sinistra). Palpo, v. fig. 2-3. Zampe senza peculiarità, non annulate. Opistosoma dorsalmente grigio chiaro con irregolari disegni scuri, ventralmente più chiaro; filiere superiori con articolo apicale bianchiccio appuntito appena più lungo del basale.

Dimensioni (in mm, leggermente approssimate): prosoma lungo 5,00, largo 3,80; opistosoma 5,20. Lunghezza totale: 10,20. 


\begin{tabular}{|c|c|c|c|c|c|c|}
\hline Zampe & Femore & Patella & Tibia & Metatarso & Tarso & Totale \\
\hline $\mathbf{I}$ & \multicolumn{6}{|c|}{ assentida ambo i lati } \\
\hline II & 7,50 & 1,80 & 7,00 & 7,50 & 3,00 & 26,80 \\
\hline III & 6,80 & 1,80 & 6,20 & 7,50 & 2,80 & 25,10 \\
\hline IV & \multicolumn{6}{|c|}{ assentida a mo i lati } \\
\hline
\end{tabular}

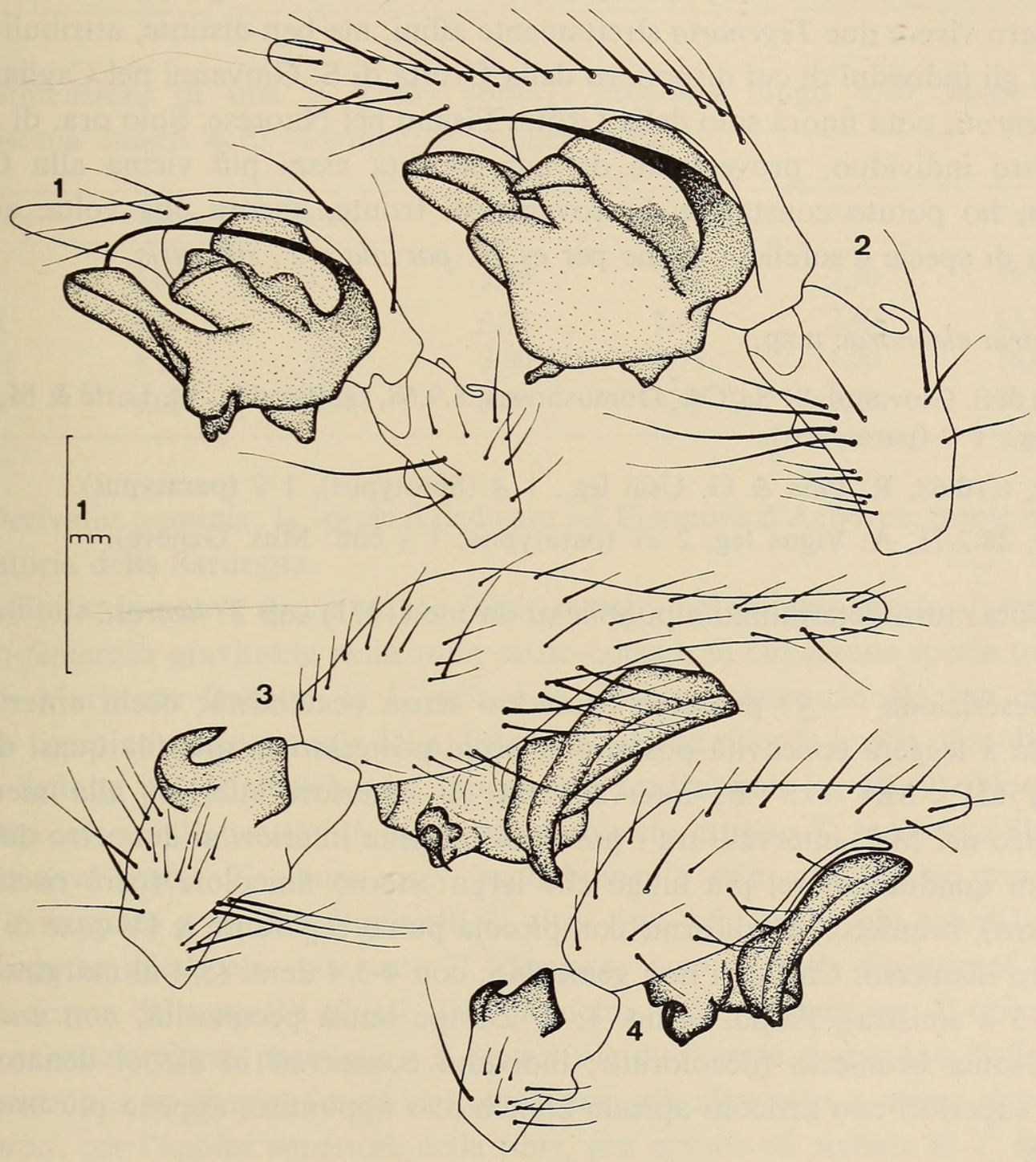

FIG. 1-4.

Tegenaria eleonorae n. sp. - Fig. 1, 4: palpo del ô. Tegenaria henroti Dresco 1956. Fig. 2, 3: palpo del ô.

Confronto con la descrizione originale: a parte piccole differenze di colore (l'individuo di Dresco era apparentemente più intensamente pigmentato), questo individuo presenta solo gli occhi LA leggermente più grandi degli altri 
(rapporto per DRESCO: LA/LP/MP > MA) ed i cheliceri hanno apparentemente ambedue 5-4 denti. Quanto a dimensioni, il «mio » 0 è più piccolo (larghezza del prosoma 3,80 contro 4,61). Dal confronto delle figure si può notare che in

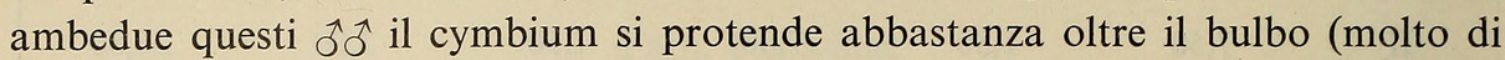
più che in T. eleonorae n.sp.); vi sono, è vero, piccole differenze nella forma del conductor, dell'apofisi basale e delle apofisi tibiali, ma nettamente minori di quelle rispetto a $T$. eleonorae n.sp.

Discussione: a suo tempo, non sospettando che in Sardegna, a breve distanza potessero vivere due Tegenaria strettamente affini, ma ben distinte, attribuii senza esitare gli individui di cui disponevo della Grotta di S. Giovanni nel Cagliaritano a $T$. henroti, nota finora solo della Grotta Pisanu nel Nuorese. Solo ora, di fronte a questo individuo, proveniente da una località assai più vicina alla Grotta Pisanu, ho potuto constatare di trovarmi di fronte, ancora una volta, ad una coppia di specie «sorelle", come per es. $T$. parvula e $T$. silvestris.

\section{Tegenaria eleonorae n.sp.}

Grotta di S. Giovanni, 81 Sa/CA, Domusnovas, 8.9.68, G. Pirodda, M. Latte \& M. Pinna leg., 1 \& (paratypus).

Ibidem, 6.10.68, R. Pilia \& G. Usai leg., 1 ơ (holotypus), 1 क (paratypus).

Ibidem, 28.2.71, A. Vigna leg. 2 우 (paratypus; 1 ㅇ coll. Mus. Genève).

Nota: tutti determinati e pubblicati da me (1971) sub $T$. henroti.

Descrizione - $\hat{\jmath}$ : prosoma bruniccio senza peculiarità; occhi anteriori in una fila a leggera concavità posteriore, occhi posteriori in una fila quasi diritta; $\mathrm{LA} / \mathrm{LP} / \mathrm{MP}: \mathrm{MA}=8: 6$; intervalli tra gli anteriori inferiori alla metà del diametro dei MA, intervalli tra i posteriori appena inferiori al diametro dei MA. Labium quadrangolare, più lungo che largo; sterno unicolore (però rischiarato al centro), bruniccio, cordiforme con piccola punta separante le IV coxe di meno del loro diametro. Cheliceri non genicolati, con 4-3/4 denti (3/4 al margine superiore, 3 a sinistra). Palpo, v. fig. 1, 4. Zampe senza peculiarità, non annulate. Opistosoma bruniccio (decolorato; individui conservati in alcool denaturato); filiere superiori con articolo apicale bianchiccio appuntito, appena più breve del basale.

O (stessa data del $\hat{\jmath}$ holotypus): meglio colorata, con tracce di disegni scuri sul prosoma, opistosoma bianchiccio con disegni irregolari neri, zampe annulate. Epigino e vulva (v. BRIGNOLI, 1971, fig. 11-12); epigino sotto forma di un'ampia piastrina trapezoidale, liscia, leggermente incavata nel mezzo nella porzione anteriore, più elevata verso il solco epigastrico, lati scuri (spermateche per trasparenza); vulva con dotti di copulazione ampi, spiralati, terminanti senza costrizioni o dilatazioni nelle spermateche, piccole e non distinte dai dotti. 
Dimensioni (in $\mathrm{mm}$, leggermente approssimate; $\hat{o}$ holotypus): prosoma lungo 3,57, largo 3,05; opistosoma lungo 4,75. Lunghezza totale: 8,32.

\begin{tabular}{|ccccccc|}
\hline Zampe & Femore & Patella & Tibia & Metatarso & Tarso & Totale \\
\hline I & 5,65 & 1,70 & 5,75 & 6,30 & 3,00 & 22,40 \\
II & 5,30 & 1,60 & 5,30 & 5,90 & 2,50 & 20,60 \\
III & 5,00 & 1,40 & 4,50 & 5,65 & 2,15 & 18,70 \\
IV & 5,50 & 1,50 & 5,75 & 7,25 & 2,75 & 22,75 \\
\hline
\end{tabular}

Dimensioni di una + (come sopra): prosoma lungo 3,70, largo 2,90; opistosoma lungo 4,10. Lunghezza totale: 7,80 .

\begin{tabular}{|ccccccc|}
\hline Zampe & Femore & Patella & Tibia & Metatarso & Tarso & Totale \\
\hline I & 5,00 & 1,50 & 5,00 & 5,50 & 2,50 & 19,50 \\
II & 4,65 & 1,40 & 4,35 & 5,10 & 2,40 & 17,90 \\
III & 4,15 & 1,35 & 3,90 & 4,75 & 1,90 & 16,05 \\
IV & 5,00 & 1,40 & 5,00 & 6,40 & 2,25 & 20,05 \\
\hline
\end{tabular}

Derivatio nominis: la specie è dedicata ad Eleonora d'Arborea, nome celebre nella storia della Sardegna.

Affinità: la nuova specie va senz'altro inserita nel gruppo ligurica-tyrrhenicahenroti-femoralis gravitante nella zona sardo-corsa e di cui alcune specie toccano le Alpi Marittime francesi, la Liguria e la Toscana settentrionale; un classico gruppo tirrenico settentrionale dalla distribuzione che ricorda quella di moltissime specie definite paleotirreniche dai biogeografi tradizionali (v. fig. 9). Non sono del tutto certo della possibilità di inserire in questo gruppo T. soriculata Simon e l'altra nuova specie, $T$. cyrnea $\mathrm{n}$.sp.; probabilmente affini, ma con un conductor meno sviluppato e genitali femminili di altro tipo (cfr. anche più avanti).

Rispetto alle specie già note $T$. eleonorae n.sp. si può distinguere per la diversa forma delle apofisi tibiali, del conductor, del cymbium ecc. È chiaro che essa è particolarmente prossima a $T$. henroti; le due specie si possono distinguere facilmente per la diversa forma del cymbium, più allongato e meno elevato in $T$. henroti, per l'apofisi superiore della tibia, più grande ed arcuata in $T$. henroti, nonché per la forma del conductor e dell'apofisi basale.

Per un confronto con le altre specie affini, v. BRIGNOLI, 1971, per T. tyrrhenica; KraUs (1955) per T. femoralis e SimON (1937) per T. ligurica.

Di Sardegna è nota anche $T$. drescoi Brignoli, 1971, di cui non è ancora conosciuto il $\widehat{o}$; poichè però questa specie per l'insieme dei caratteri va senz'altro inserita nel gruppo nemorosa, non è possibile che essa corrisponda a $T$. eleonorae n.sp. 


\section{Tegenaria sp.}

Grotta Verde, 3 Sa/SS, Alghero, 18.3.71, V. Aellen \& P. Strinati leg., 1 ○.

Immaturo indeterminabile (nessuna Tegenaria era nota della $3 \mathrm{Sa} / \mathrm{SS}$ ).

\section{MATERIALE DI CORSICA}

Tegenaria cyrnea n.sp.

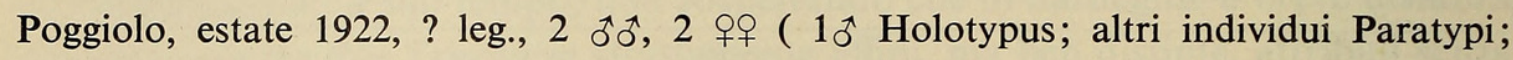

N.B.: determinati - da A. Comellini ? - come T. soriculata Simon 1873).

Descrizione - $\widehat{\jmath}$ : prosoma bruno rossiccio, senza peculiarità; occhi anteriori in una fila a leggerissima concavità posteriore, occhi posteriori in una fila diritta;

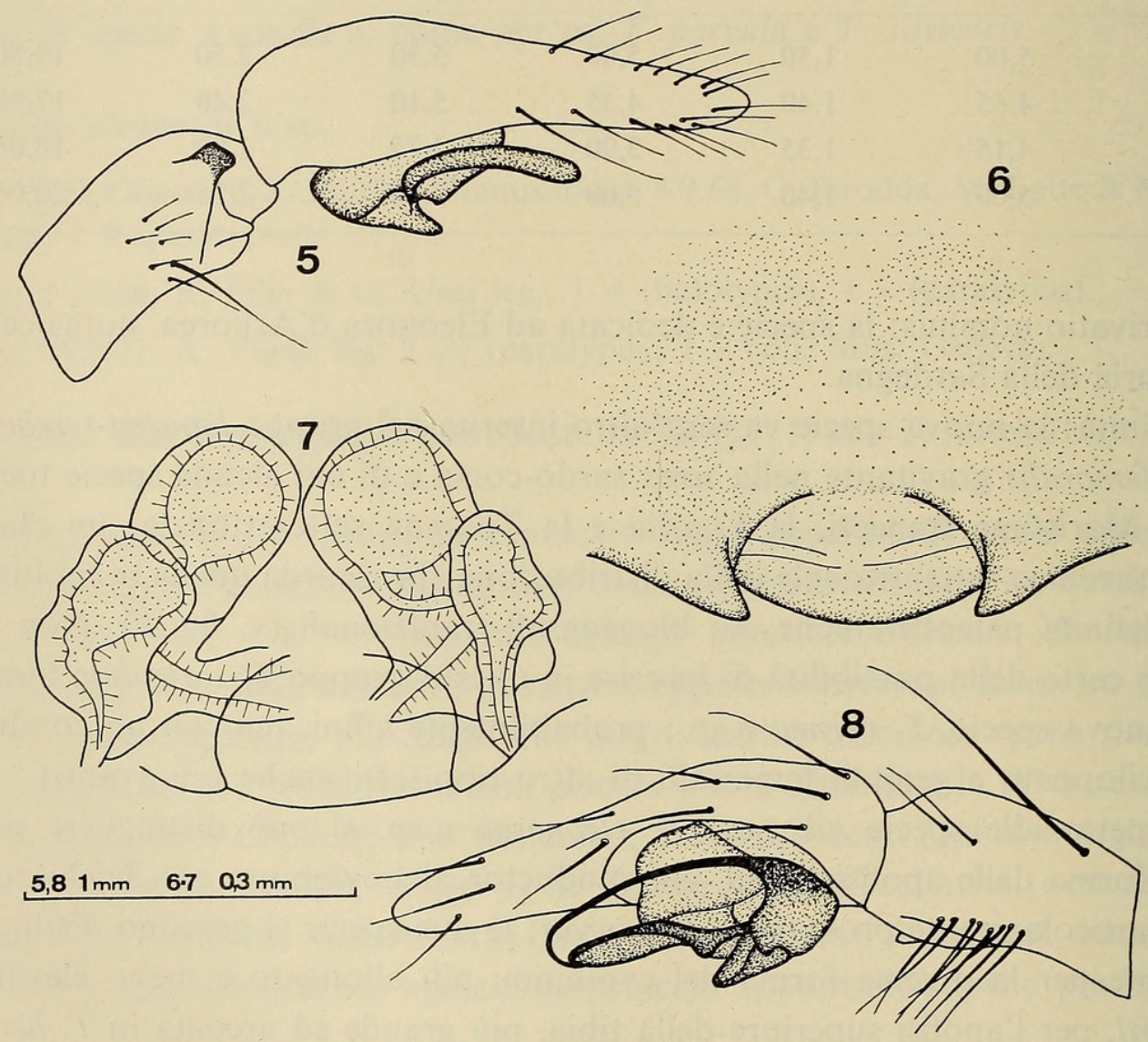

FIG. 5-8.

Tegenaria cyrnea $\mathrm{n} . \mathrm{sp}$.

Fig. 5, 8: palpo del ô; 6: epigino; 7: vulva.

occhi adeguali (MA appena più piccoli degli altri, $5: 7$ ), anteriori ravvicinati (intervalli pari alla metà del diametro dei MA), posteriori più distanziati (intervalli quasi pari al diametro dei MA). Labium quadrangolare, più lungo che largo; 
sterno unicolore, bruniccio, cordiforme con piccolissima punta separante le IV coxe di molto meno del loro diametro. Cheliceri rossicci, non genicolati, con 4-3 denti (4 al margine inferiore). Palpo, v. fig. 5, 8, simile a quello di $T$. soriculata, ma distinguibile per numerosi caratteri. Zampe unicolori, giallicce. Opistosoma (molto rovinato in tutti gli individui) ardesia scuro, più chiaro ventralmente; filiere superiori con articolo basale cilindrico, rimbrunito, nettamente più corto dell'apicale che è bianchiccio appuntito.

q: corrispondente al $\widehat{\partial}$, cheliceri però genicolati. Epigino e vulva, v. fig. 6-7.

Dimensioni (in $\mathrm{mm}$, leggermente approssimate; $\hat{o}$ holotypus): prosoma lungo 4,80, largo 4,20; opistosoma (contratto) lungo 3,20. Lunghezza totale (attuale): 8,00 .

\begin{tabular}{|ccccccc|}
\hline Zampe & Femore & Patella & Tibia & Metatarso & Tarso & Totale \\
\hline I & 3,50 & 1,60 & 3,00 & 3,20 & 2,00 & 13,30 \\
II & 3,00 & 1,25 & 2,75 & 3,12 & 2,00 & 12,12 \\
III & 3,00 & 1,12 & 2,12 & 3,00 & 2,00 & 11,24 \\
IV & 3,87 & 1,62 & 3,67 & 4,12 & 2,12 & 15,40 \\
\hline
\end{tabular}

Dimensioni di una $ᄋ$ (come sopra): prosoma lungo 4,00, largo 3,50; opistosoma (contratto) lungo 3,80. Lunghezza totale (attuale): 7,80.

\begin{tabular}{|ccccccc|}
\hline Zampe & Femore & Patella & Tibia & Metatarso & Tarso & Totale \\
\hline I & 2,95 & 1,37 & 2,17 & 2,25 & 1,50 & 10,24 \\
II & 2,50 & 1,20 & 1,92 & 2,20 & 1,35 & 9,17 \\
III & 2,50 & 1,00 & 1,75 & 2,12 & 1,00 & 8,37 \\
IV & 3,00 & 1,25 & 2,37 & 3,25 & 1,25 & 11,12 \\
\hline
\end{tabular}

Derivatio nominis: « cyrnea » deriva dal nome greco della Corsica.

Affinità: la nuova specie è senz'altro affine a $T$. soriculata Simon, 1873 , nota di Corsica e Sardegna (su questi ultimi reperti, dovuti a GARNERI, 1902, e a KraUS, 1955, nutro qualche dubbio), ma ne è distinguibile per la forma delle apofisi tibiali, del bulbo e dell'epigino. È interessante notare che la vulva di questa specie, il cui $\hat{o}$ sembra prossimo al gruppo ligurica, è invece alquanto differente da quella delle $\bigcirc \bigcirc$ di questo gruppo e può ricordare i gruppi pagana o agrestis. 


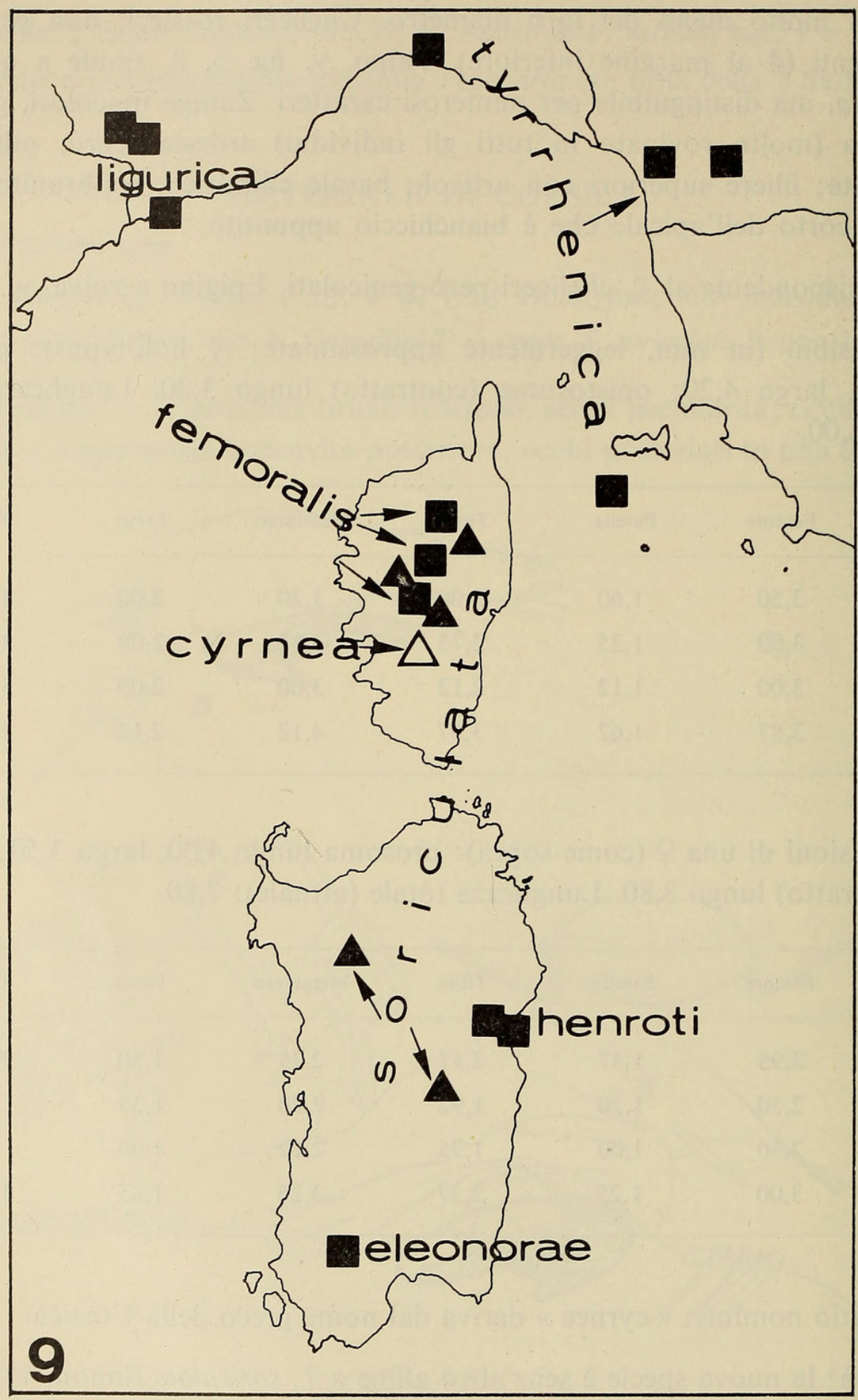

FIG. 9.

Distribuzione delle Tegenaria dei gruppi ligurica (quadrati neri) e soriculata (triangoli); per l'elenco delle stazioni v. Brignoli, 1971, e KraUs, 1955. N.B.: mi è ignota l'esatta ubicazione di Poggiolo (unica stazione nota di $T$. cyrnea) e di Monte Nuovo in Sardegna (stazione di T. soriculata secondo GARNERI, 1902). 


\section{SUMMARY}

Some species collected in three Sardinian caves are listed; Tegenaria henroti 1956, found in a second locality, is redescribed $(\widetilde{0})$. The species identified by BRIGNOLI (1971) as T. henroti is here described as new: T. eleonorae n.sp. ( $\hat{t} q$; typ. loc.: S. Giovanni cave, near Domusnovas); this species belongs to the liguricagroup and can be distinguished from the other related species by the morphology of the genitalia. From Corsica is described Tegenaria cyrnea n.sp.: $\uparrow+$, near to T. soriculata Simon, 1873, distinguishable by the genitalia of both sexes; typ. loc.: Poggiolo; these two species form a small group somewhat near to the liguricagroup.

\section{ZUSAMMENFASSUNG}

Einige Arten, die in drei Höhlen Sardiniens gesammelt wurden, werden gemeldet. Tegenaria henroti Dresco, 1956, wurde zum zweiten Male gefunden und nach einem $\hat{\sigma}$ wiederbeschrieben. Die Art, die von BrignOLI (1971) als T. henroti identifiziert wurde, wird als neue Art, T. eleonorae n.sp. ( $₫$ 웅 locus typicus: die Hohle S. Giovanni bei Domusnovas) beschrieben. Diese Art gehört zur ligurica-Gruppe und kann von den anderen verwandten Arten genitalmorphologisch unterschieden werden. Von Korsika wird Tegenaria cyrnea n.sp. ( $\hat{\sigma}^{\circ}$ ) beschrieben, die $T$. soriculata Simon, 1873, nahe steht, jedoch in beiden Geschlechtern genitalmorphologisch unterschieden werden kann (locus typicus: Poggiolo). Diese beiden Arten bilden eine kleine Gruppe, die etwas der liguricaGruppe nahe steht.

\section{BIBLIOGRAFIA}

BRIGnoli, P. M. 1969. Secondo contributo alla conoscenza dei Leptonetidae della Sardegna. Archo. Zool. ital. 54: 11-31.

Brignoli, P. M. 1971. Contributo alla conoscenza degli Agelenidae italiani. Fragm. ent. 8: 57-142.

Brignoli, P. M. 1972. Catalogo dei ragni cavernicoli italiani. Quad. Speleol. Circ. Speleol. Rom. 1: 5-212.

Dresco, E. 1956. Tegenaria henroti, espèce nouvelle des grottes de Sardaigne Fragm. ent. 2: 115-119.

Garneri, G. A. 1902. Contribuzione alla fauna sarda. Aracnidi. Boll. Soc. zool. ital. (2) $3: 57-103$.

Kraus, O. 1955. Spinnen aus Korsika, Sardinien und Elba. Senckenberg. biol. 36: 371-394.

Simon, E. 1937. Les arachnides de France. Paris 6 (5): 979-1298.

Adresse de l'auteur :

Istituto di Zoologia

Viale dell'Universita 32

I-00100 Roma

Italie 


\section{$2 \mathrm{BHL}$ Biodiversity Heritage Library}

Brignoli, Paolo Marcello. 1974. "Ragni d'Italia XXI. Settimo contributo alla conoscenza der ragni cavernicoli di Sardegna e descrizione di una nuova specie di Corsica (Araneae)." Revue suisse de zoologie 81, 387-395. https://doi.org/10.5962/bhl.part.76010.

View This Item Online: https://www.biodiversitylibrary.org/item/126812

DOI: https://doi.org/10.5962/bhl.part.76010

Permalink: https://www.biodiversitylibrary.org/partpdf/76010

\section{Holding Institution}

Smithsonian Libraries

\section{Sponsored by}

Biodiversity Heritage Library

\section{Copyright \& Reuse}

Copyright Status: In Copyright. Digitized with the permission of the rights holder.

Rights Holder: Muséum d'histoire naturelle - Ville de Genève License: http://creativecommons.org/licenses/by-nc-sa/3.0/ Rights: https://www.biodiversitylibrary.org/permissions/

This document was created from content at the Biodiversity Heritage Library, the world's largest open access digital library for biodiversity literature and archives. Visit BHL at https://www.biodiversitylibrary.org. 